\title{
Introduction to Evidence Based Medicine
}

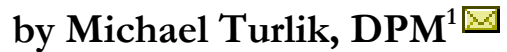

The Foot \& Ankle Journal 2 (2): 4

This paper provides an introduction to evidence-based medicine for physicians. Evidence-based medicine is defined, a historical perspective is presented and the levels of evidence are explained. Using a clinical scenario a foreground question is developed and a MEDLINE search is performed to locate the best evidence pertaining to the foreground question. A series of articles will be presented in future issues of this journal critically evaluating selected publications identified during the MEDLINE search.

Key words: EBM, evidence-based medicine, foreground question, PICO method.

Accepted: January, 2009

Published: February, 2009

This is an Open Access article distributed under the terms of the Creative Commons Attribution License. It permits unrestricted use, distribution, and reproduction in any medium, provided the original work is properly cited. @The Foot \& Ankle Journal (www.faoj.org)

\section{Introduction}

I was fortunate enough to recently attend an alternative medicine seminar. One of the speakers was a $\mathrm{PhD}$ advocating the use of magnets in the treatment of various medical conditions. In an articulate and professional manner, she explained the pathophysiology of the disease with reference to electromagnetism and provided a logical explanation of why magnets should work. Specifically she advocated using magnetic insoles for the symptomatic relief of diabetic neuropathy. She presented a convincing series of cases involving patients with diabetic neuropathy who responded to the use of magnetic insoles. Over $90 \%$ of the patients related symptomatic relief of their painful disability.

Address correspondence to: Michael Turlik, DPM

Email: mat@evidencebasedpodiatricmedicine.com

${ }^{1}$ Private practice, Macedonia, Ohio.
In addition, she stated that she has patented a new type of magnet which is superior to older versions of therapeutic magnets. This new "polarizing" magnet was on sale in the lobby at the conclusion of the talk for those practitioners interested in dispensing them to their patients. The talk met with universal acceptance by the practitioners in attendance, many of whom remarked that they have been using "polarizing" magnets in their practices for years with "good results". It is estimated that the general public spends more than $\$ 1$ billion a year purchasing magnets for the treatment of disease. ${ }^{1}$ Despite the speakers' professionalism and the audience's response, I remained somewhat skeptical about the use of magnets in the treatment of diabetic neuropathy. 
This skepticism is based on a number of questions and issues. For example, how effective are the various treatment plans which we use on a daily basis for our patients? Even more confusing might be the use of diagnostic studies in the workup of our patients, when are they indicated and how well do they help us in making a diagnosis? Answers to these and other healthcare questions require information. Information regarding patient care comes from various sources to include: anecdotal information from experts, textbooks, internet searches, continuing medical education, published articles, drug and device manufacturing representatives. How to place this information or evidence in a clinical context is what a large part of evidence-based medicine is about.

\section{Evidence-based medicine}

Evidence-based medicine can be defined as the conscientious, explicit, judicious use of the current best evidence combined with clinical expertise and patient values in making decisions about the care of individual patients. ${ }^{2}$ It is a structured approach to literature evaluation, leading to decisions which are based upon probability. The true effect of an intervention is never known for certain and we can only estimate where the truth may lie. In other words, the better the study, the closer we get to the truth.

In order to utilize evidence-based medicine in clinical practice it requires a paradigm shift from unsystematic, anecdotal observations, common sense, and pathophysiological rationales to the critical evaluation of medical information. In order to accomplish this evidence-based medicine requires the development of a new set of skills including foreground question generation, efficient literature searching resulting in the retrieval of the best evidence and the application of formal rules of evidence in evaluating the study methods to ascertain the validity of results.
Steps in Evidence-based medicine

- Develop foreground question from patient information using the PICO* method.

- Using the question developed and perform a search to locate the best evidence available.

- Critically appraise the evidence located for validity (internal/external).

- Integrate the information found with clinical expertise, patient values and apply in clinical practice.

Figure 1 Step development in evidence based medicine. $(* \mathrm{PICO}=$ Patient/Population/Problem, Intervention/Exposure, Comparison and Outcome)

Once valid and relevant clinical studies have been obtained, evidence-based medicine then requires the communication of the benefits, harms, costs, and inconveniences to patients in order to make decisions for individual patients based upon their perspectives, beliefs, expectations and values.

(Fig. 1)

\section{History of evidence-based medicine}

Prior to the 1950s, health care decisions were based primarily on anecdotal information, pathophysiology and the expert opinions of leaders in the profession. Randomized controlled trials were introduced into medicine near the end of the 1950s. During the 1980s a Canadian physician, Dr. David Sackett, began to develop a system for the critical analysis of the medical literature. His innovations proved to be a seminal event in the development of evidence-based medicine. In the early part of the 1990s, Dr. Archie Cochrane, a British physician, began advocating the use of systematic reviews in medicine. The current Cochrane collaboration is his legacy. The actual term evidence-based medicine is credited to Dr. Gordon Guyatt from McMaster University whose pioneering work appeared in the Journal of the American Medical Association as a series of publications entitled the " users' guide series" in the 1990's. 
Levels of evidence for therapeutic interventions $^{3}$

Evidence-based medicine seeks to prioritize information in a hierarchy of evidence by study design from the most biased to the least biased. For example, the use of magnetic insoles in the treatment of diabetic neuropathy would be classified as a therapeutic intervention. Therefore the highest study design would be randomized controlled trials, systematic reviews and metaanalysis. This would be considered level I evidence whereas, unsubstantiated anecdotal information would be considered level $\mathrm{V}$ or most biased therefore least useful. Evidence levels vary with the type of question to be evaluated. For example, therapy, prognosis, diagnosis questions all vary with regards to the best study design.

\section{Level $V$}

Unsystematic anecdotal information is considered the least valid form of evidence regarding therapeutic interventions. Anecdotal information is best used to develop hypotheses which are tested using more rigorous study designs. Although anecdotal information is extremely popular in the medical profession it is highly biased and impossible to verify therefore, it is of limited usefulness in determining treatment efficacy. The sources of anecdotal information may include any of the following: experts in the field, colleagues, drug and device manufacturers. Other examples of level $\mathrm{V}$ evidence include cadaver studies and animal studies.

\section{Level IV}

Case reports and series are extremely popular in the medical literature. ${ }^{4}$ They are easy to report and inexpensive. They are usually retrospective but may be prospective. Again, case studies and case series are best utilized to develop hypotheses rather than test hypotheses. They lack a control group, and suffer from selection bias. It does not matter how many subjects were enrolled or how long the study was carried forward.
Case reports often overestimate treatment effects. For example, Mullen, et al., in a series of cases over eight years reported good results using Cimetidine for the treatment of plantar warts in children. However, when this treatment was reviewed in two randomized controlled trials, Cimetidine was shown to be ineffective. ${ }^{5}$ If caseseries are sufficiently large they may be very useful in documenting adverse effects of treatments which have been shown to be effective.

Level III

Case-control studies are a type of observational study in which the outcome has already occurred. These are retrospective studies using a comparator. These types of studies are best used to evaluate rare conditions. The results of these studies are usually expressed as odds ratios. It is difficult to find a good control group for these studies therefore; selection bias is always a concern. Recall bias is another common concern regarding case-control studies. These types of studies are usually not very common in the podiatric literature. ${ }^{4}$

\section{Level II}

Cohort studies are another type of observational comparative study which begins with an exposure rather than outcome. Usually these studies are prospective, but maybe retrospective. This type of study (prospective) tends to minimize recall bias. These studies are expensive, time-consuming and require careful attention to detail in evaluating the control group. Regardless of the care taken to choose the control group, selection bias is always a concern. Results typically are expressed as a relative risk rather than odds ratios. These types of studies are not common in the podiatric literature. $^{4} \quad$ Cohort studies should not be confused with the term cohort. Cohort refers to a group of patients, cohort studies are a type of observational study using a comparator.

(C) The Foot \& Ankle Journal, 2009 


\section{Level I}

Randomized controlled trials unlike the preceding study designs are true controlled experiments. Two or more groups of subjects receive different interventions and are followed forward in time and at some point they are compared using an outcome. This type of study design minimizes selection bias however, despite the fact that the concept is simple they are difficult to perform correctly and incur considerable expense. Although not common in the podiatric literature ${ }^{4,6}$, there has been an increase in the number of these types of clinical trials in medicine over the last 10 years. Although extremely common for non-surgical treatments only recently have they gained recognition and acceptance in surgical sub specialties. ${ }^{7,8}$

Systematic reviews are a special type of review article which can be considered level I evidence when randomized controlled trials are utilized. Systematic reviews, unlike textbooks or narrative reviews, require careful planning and methods which minimize bias and random error. Like any well performed study the methods are transparent and allow other researchers to replicate the results and reach similar conclusions. Meta-analysis is a specialized type of systematic review which pool data for a quantitative rather than a qualitative result. They also can be considered level I evidence if the study is limited to randomized controlled trials.

Even though randomized controlled trials are considered level I evidence, the believability of the results varies with the rigor with which the study was performed. In a recent meta-analysis performed by Thomson evaluating the use of extracorporeal shock wave therapy for plantar heel pain, he found that when the results of the two poorest quality randomized controlled trials were removed the results of the meta-analysis was no longer statistically significant. ${ }^{9}$ Lesser levels of evidence or poorly designed and executed studies overestimate treatment effects.

\section{Clinical Scenario: Magnets and diabetic neuropathy}

If we follow the step develop in evidence based medicine: A foreground question ${ }^{10}$ could include; "In diabetics with symptomatic distal sensory neuropathology are magnetic insoles effective in reducing the painful sensations of diabetic neuropathy in diabetic patients?" When searching for the best evidence the podiatric physician has a choice between pre-appraised research and original research.

Pre-appraised research is the wave of the future since busy clinicians have little time and may lack the skills necessary to critically evaluate the medical literature. The Cochrane collaboration ${ }^{11}$ is an example of a pre-appraised, searchable database. There are many others available. The pre-appraised sites are usually by subscription and mostly pertain to primary care medicine.

MEDLINE is the classic searchable medical database for original research. Using the foreground question developed regarding magnets and diabetic neuropathy, a literature search was performed using MEDLINE limiting the results to randomized controlled trials, and meta-analysis in an attempt to locate potential sources of level I evidence. Using the keywords magnets, pain, diabetic neuropathy, feet, the Boolean operator AND various results were returned.(Table 1) One of the results included a meta-analysis of randomized controlled trials which evaluated the use of magnets in reducing pain. ${ }^{1}$

Reviewing the references from the meta-analysis a randomized controlled trial evaluating the use of magnetic insoles for the treatment of diabetic neuropathology ${ }^{12}$ was found. It should be noted that level I evidence may not always be found. Relying on lesser levels of evidence reduces the inference drawn regarding the therapeutic option. 


\begin{tabular}{|lc|}
\hline Search term & Results \\
\hline Magnets & 34 \\
\hline Magnets AND pain & 18 \\
\hline Magnets AND feet & 2 \\
\hline Magnets AND diabetic neuropathy & 0 \\
\hline
\end{tabular}

Table 1 Results of MEDLINE search

Once potential sources of level I evidence are located the physician needs to answer the three following questions to critically appraise the articles found:

1. Have the author(s) reasonably attempted to limit bias.? ( Internal validity)

2. What are the results?

3. Will the results of the study help me in caring for my patient? (External validity)

Future articles in this series will provide the answers to these questions using the references identified $^{1,12}$ in the MEDLINE search for magnets and diabetic neuropathy.

\section{References}

1. Pittler MH, Brown EM. Static magnets for reducing pain: systematic review and meta-analysis of randomized trials. CMAJ 177 (7): 736 - 742, 2007.

2. Sackett DL, Rosenberg WM, Gray JA, Haynes RB, Richardson WS. Evidence based medicine: what it is and what it isn't. BMJ 312 (7023): 71 - 2, 1996.

3. Levels of Evidence, [Online-accessed 1/10/2009].

4. Turlik M, Kushner D. Levels of evidence of articles in podiatric medical journals. JAPMA 90 (6): 300 - 302, 2000. 5. Mullen BR, Guiliana JV, Nesheiwat F. Cimetidine as a first-line therapy for pedal verruca. JAPMA 95 (3): 229 234, 2005.

6. Turlik M, Kushner, D, Stock, D. Assessing the validity of published randomized controlled trials in podiatric medical journals. JAPMA 93 (5): 392 - 398, 2003.

7. Obremskey WT, Pappas N, Attallah-Wasif E, Tornetta P, Bhandari M. Level of evidence in orthopedic journals. . Journ Bone Joint Surg, 87A (12): 2632 - 2638, 2005.

8. MaierV. What the surgeon of tomorrow needs to know about evidence-based surgery. Arch Surg, 141: 317 - 323, 2006.

9. Thompson C, Crawford F, Murray GD. The effectiveness of extra corporeal shock wave therapy for plantar heel pain: a systematic review and meta-analysis. BMC Musculoskeletal Disorders 6 (19), 2005.

10. Weinfeild JM, Finkelstein K. How to answer your clinical questions more efficiently. [Online-accessed $1 / 10 / 09]$.

11. The Cochrane collaboration, [Online-accessed $1 / 10 / 09]$.

12. Weintraub MI, Wolfe GI, Barohn RA, Cole SP, Parry GJ, Hayat G, Cohen JA, Page JC, Bromberg MB, Schwartz SL, Magnetic Research Group. Static magnetic field therapy for symptomatic diabetic neuropathy: a randomised, doubleblind, placebo-controlled trial. Arch Phys Med Rehab 84: 736 - 46, 2003. 